\title{
Currency lookback options and observation frequency: a binomial approach
}

\author{
TERRY H F CHEUK \\ Department of Finance, Erasmus University, Rotterdam and Tinbergen \\ Institute, $3000 \mathrm{DR}$, Rotterdam, Netherlands \\ AND \\ TON C F VORST* \\ Department of Finance, Erasmus University, Rotterdam and Erasmus \\ Center for Financial Research, 3000 DR, Rotterdam, Netherlands
}

\begin{abstract}
In the last decade, interest in exotic options has been growing, especially in the over-the-counter currency market. In this paper we consider lookback currency options, which are path-dependent. We show that a one-state variable binomial model for currency lookback options can be constructed with the same computational complexity, or should we say simplicity, as the standard binomial model. Furthermore, the model allows us to investigate a second very important feature of real-life lookback option contracts - the observation frequency. (JEL F31). (C) 1997 Elsevier Science Ltd.
\end{abstract}

The binomial option model introduced by Cox et al. (1979) has been instrumental in option pricing theory and practice for two reasons - simplicity and flexibility. First, its simple framework allows a straightforward derivation of the option pricing results of Black and Scholes (1973) and illustrates the fundamental insight of Cox and Ross (1976) that derivative securities should be priced within a risk-neutral economy. Their approach uses simple calculus, without the need of the complicated mathematical theory of stochastic Brownian motions. Second, it gives a fast and numerically accurate method to price American options. For these two reasons the binomial model has become popular with practitioners and academics alike.

In the last decade, interest in exotic options has been growing, especially in the over-the-counter currency market. For internationally operating firms with

\footnotetext{
${ }^{*}$ Corresponding author.
} 
complicated cashflow patterns involving various currencies, derivative products are constructed according to their specific needs for hedging. A lot of research has been devoted to the pricing and hedging of these products, not only within the continuous-time framework of Black and Scholes, but increasingly also within the discrete-time economy of Cox, Ross and Rubinstein. This latter development is due to the previously described benefits of the binomial model. However, the feasibility of a discrete model depends heavily on the number of nodes involved. Thus, the usefulness of a discrete model for a specific kind of option contract depends not only on the numerical accuracy, but also on the computer time needed for the calculations.

An option is called path-dependent if its price depends on the path followed by the underlying asset since the inception of the contract, in addition to the underlying asset price and the remaining time to maturity. Usually, there exists one sufficient statistic describing the past. For example, for an average rate (or Asian) currency option, which is popular with firms that have a stream of cashflows in a foreign currency, this statistic is the average of the past values of the exchange rate. The sufficient statistic can be added as a second state variable to the model. However, it is well known that binomial models with two state variables converge slowly to the limiting value.

In this paper we consider lookback currency options, which are path-dependent. For these options the sufficient statistic is either the minimum of the underlying exchange rate since the inception of the contract, or the maximum. Hull and White (1993) have developed a binomial model for these options, in which each node of the binomial tree is subdivided into different states, according to the value of the sufficient statistic. Hence, they have an implicit two-state variable model. We show that a one-state variable binomial model for currency lookback options can be constructed with the same computational complexity, or should we say simplicity, as the standard binomial model. Furthermore, the model allows us to investigate a second very important feature of real-life lookback option contracts - the observation frequency.

Analytical pricing formulas for lookback options have been derived by Goldman et al. (1979) and were rederived and extended by Conze and Viswanathan (1991). These formulas are based on the continuous-time assumptions of the Black-Scholes economy. Especially, it is assumed that the minimum or maximum is taken over the continuous-time path of the exchange rate. However, in real-life contracts the minimum or maximum is calculated over a prespecified number of fixings only, such as daily, weekly or monthly. Hence, every contract has a fixed, discrete number of observations. We show that this feature can be incorporated into the binomial model, which is not possible in the analytic approach, just as the Cox, Ross and Rubinstein model enables us to price American options, while the analytical Black-Scholes approach does not allow early exercise. More importantly, we show that the number of fixings is a very significant variable. For instance, there are large price differences between contracts based on weekly and daily fixings. Furthermore, even with daily fixings, values of lookbacks differ significantly from those calculated with analytical models.

Lookback options can be divided into two classes: floating strike and fixed 
strike. A European floating strike currency lookback call option, or simply a European currency lookback call option, gives the holder the right to buy the underlying currency at maturity for the lowest exchange rate over the observation dates. A European floating strike currency lookback put option, or simply a European currency lookback put option, allows the holder to sell the underlying currency for the highest exchange rate over the observation dates. A European fixed strike currency lookback call option entitles the holder to the difference between the highest value of the underlying over the observation dates and the strike price, as long as this number is positive. A European fixed strike currency lookback put option pays the difference between the strike price and the minimum value over the observations, if it is positive. Fixed strike lookbacks are sometimes called options on extrema (see Conze and Viswanathan, 1991). We derive different models for fixed and floating strike currency lookbacks. In this paper we focus on currency options, since lookback options are mostly structured with a foreign exchange rate as an underlying variable. These options are popular with internationally operating firms. As far as we know the only other underlying variables for lookback options are indexes. However, our formulas can be used in this case too, if one replaces the foreign interest rate with the dividend yield on the index.

Babbs (1992) has also derived a binomial model for floating strike lookback options. However, his model is based on the continuous-time Black-Scholes economy and uses the stochastic theory of Brownian motions. Our model only uses the arbitrage arguments of the Cox, Ross and Rubinstein model, i.e. simple calculus. For discretely observed lookback options both methods have the same computational efficiency. We compare our approach to that of Babbs (1992) and the method by Hull and White (1993) in Section IV.

This paper is organized as follows. In the next section the basic binomial models for floating strike and fixed strike currency lookback options are derived. We assume every point in the tree is an observation date. In Section II this assumption will be relaxed, and we are able to discover the price differences between weekly, daily and continuous observations. Section III considers American options. Section IV compares our approach to alternative methods, while the final section concludes the paper.

\section{The binomial models}

In this section, valuation models for currency lookback options are described. The familiar Cox et al. (1979) binomial model, as described in Figure 1, is used as a general framework for pricing.

If $T$ is the time to maturity of the option and $N$ is the number of discrete time points, we have time points $t_{0}, t_{1}, t_{2}, \ldots, t_{N}$, with $t_{i}=i T / N$. As usual, we assume $u=e^{\sigma \sqrt{\Delta t}}$ and $d=1 / u$, where $\Delta t=T / N$ is the length of the time interval between two discrete time points, and $\sigma$ is the underlying exchange rate volatility. Let $r$ be one plus the risk-free domestic interest rate over a discrete-time period, i.e. $r=e^{r_{d} \Delta t}$, where $\bar{r}_{d}$ is the continuous-time riskfree domestic rate. $\tilde{r}_{f}$ is the riskfree foreign rate, $\mu$ is the no-arbitrage growth rate of the currency, i.e. $\mu=e^{\left(\tilde{r}_{d}-\vec{r}_{j}\right) \Delta t}$. Since a put option on one currency is a call 


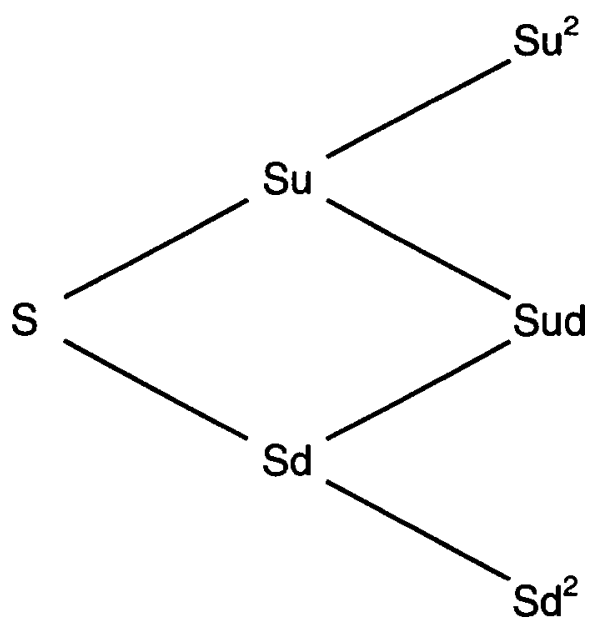

FIgURE 1. Cox, Ross and Rubinstein model.

on the other one, a separate model for currency lookback puts is not required. Following Cox et al. (1979), if $C\left(S, t_{j}\right)$ is the option price at time point $t_{j}$ with underlying stock value $S$, then

$$
C\left(S, t_{j}\right)=\left[p C\left(S u, t_{j+1}\right)+(1-p) C\left(S d, t_{j+1}\right)\right] / r
$$

with

$$
p=\frac{\mu-d}{u-d}
$$

The probabilities $p$ and $1-p$ are the so called risk-neutral probabilities. This relation also holds for the options studied in this paper, since the same option replication argument can be used. However, the value of currency lookback options depends also on a third factor, other than time and underlying exchange rate. First, consider a European floating strike currency lookback call option. The payoff at maturity of this call option is equal to

$$
S(T)-\min _{0 \leq i \leq N} S\left(t_{i}\right),
$$

where $t_{0}$ represents the starting point in time from where the minimum is taken, which is not necessarily the actual time. Assume the actual time is $t_{j}$. In addition to the actual exchange rate $S\left(t_{j}\right)$ and the remaining time to maturity, the option price also depends on

$$
\underline{M}\left(t_{j}\right)=\min _{0 \leq i \leq j} S\left(t_{i}\right)
$$

$\underline{M}\left(t_{j}\right)$ is measurable at time $t_{j}$.

Let $C\left(S\left(t_{j}\right), \underline{M}\left(t_{j}\right), t_{j}\right)$ be the price of the floating strike currency lookback call option. 
Define:

$$
k=\ln \left[S\left(t_{j}\right) / \underline{M}\left(t_{j}\right)\right] / \ln (u) .
$$

Since $\underline{M}\left(t_{j}\right)$ is a minimum over values $S\left(t_{0}\right) u^{i}$, with $i$ an integer, it follows that $k$ is a non-negative integer indicating the difference in powers of $u$, between the actual and the lowest exchange rate between time $t_{0}$ and the actual time, i.e. $k=0,1, \ldots, j$.

We claim that $C\left(S\left(t_{j}\right), M\left(t_{j}\right), t_{j}\right)$ is equal to $S\left(t_{j}\right)$ multiplied by a function $V$, which depends on $k$ and actual time $t_{j}$ only, i.e.

$$
C\left(S\left(t_{j}\right), \underline{M}\left(t_{j}\right), t_{j}\right)=S\left(t_{j}\right) V\left(k, t_{j}\right) .
$$

Hence, to determine the option price we only have to find the function $V$. Since $V$ depends on one state variable $k$ and the time variable only, we are able to construct a simple binomial model for the floating strike currency lookback call option. The claim is proved by backward induction and follows in due course. As

$$
C\left(S\left(t_{N}\right), \underline{M}\left(t_{N}\right), t_{N}\right)=S\left(t_{N}\right)-\underline{M}\left(T_{N}\right)=S\left(t_{N}\right)\left(1-u^{-k}\right),
$$

our claim holds for the maturity date, if we define

$$
V\left(k, t_{N}\right)=1-u^{-k} \text {. }
$$

Now we can construct a tree as in Fig. $2^{1}$. If $k \geq 1$ at time $t_{j}$ and the exchange rate goes upfrom $S$ to $S u$, then at time $t_{j+1}$, the difference in powers of $u$ is one larger than at time $t_{j}$, i.e. $k+1$. If the exchange rate goes down, the difference becomes $k-1$. If $k=0$ at time $t_{j}$ and the exchange rate rises, $k$ becomes 1 . However, $k=0$ indicates the actual exchange rate is equal to the minimum exchange rate so far. If the exchange rate drops further, the new exchange rate will also be the new minimum, i.e. $k=0$ at time $t_{j+1}$. This is exactly described in Figure 2. In order to determine the values of the function $V$, the well-known backward induction procedure is used.

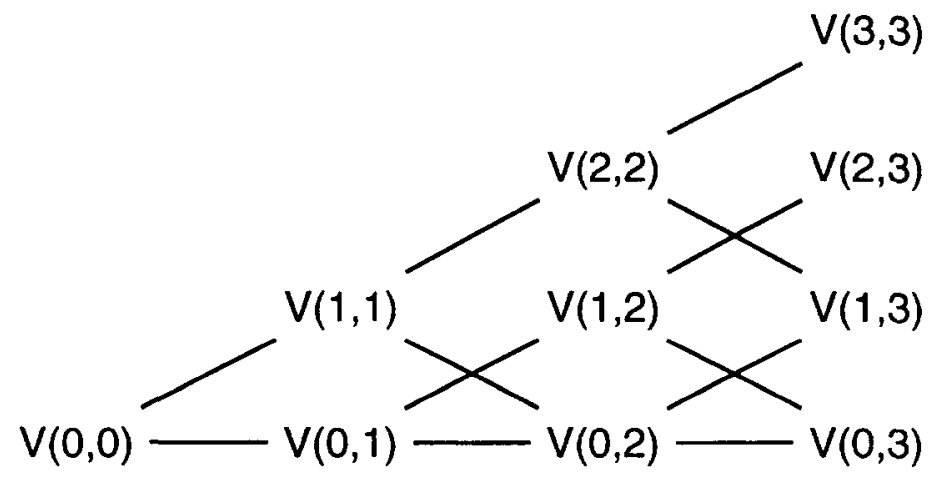

FIGURE 2. Floating strike currency lookback call. 
Consider the point $\left(k, t_{j}\right)$ with $k \geq 1$ and let the exchange rate be equal to $S\left(t_{j}\right)$. We know from the Cox, Ross and Rubinstein arbitrage argument that

$$
\begin{aligned}
C\left(S\left(t_{j}\right), \underline{M}\left(t_{j}\right), t_{j}\right)= & {\left[p C\left(S\left(t_{j}\right) u, \underline{M}\left(t_{j+1}\right), t_{j+1}\right)\right.} \\
& \left.+(1-p) C\left(S\left(t_{j}\right) d, \underline{M}\left(t_{j+1}\right), t_{j+1}\right)\right] / r,
\end{aligned}
$$

where $\underline{M}\left(t_{j+1}\right)=\underline{M}\left(t_{j}\right)$, since $k \geq 1$.

By the backward induction assumption, it follows that

$$
\begin{aligned}
C\left(S\left(t_{j}\right), \underline{M}\left(t_{j}\right), t_{j}\right)= & {\left[p S\left(t_{j}\right) u V\left(k+1, t_{j+1}\right)\right.} \\
& \left.+(1-p) S\left(t_{j}\right) d V\left(k-1, t_{j+1}\right)\right] / r
\end{aligned}
$$

Hence, if we define

$$
V\left(k, t_{j}\right)=\left[q V\left(k+1, t_{j+1}\right)+(1-q) V\left(k-1, t_{j+1}\right)\right] \frac{\mu}{r},
$$

with

$$
q=\frac{\mu u-1}{\mu(u-d)},
$$

formula $\langle 6\rangle$ follows and our claim at time $t_{j}$ is proved.

Similarly, the claim follows for $k=0$ if we define

$$
V\left(0, t_{j}\right)=\left[q V\left(1, t_{j+1}\right)+(1-q) V\left(0, t_{j+1}\right)\right] \frac{\mu}{r} .
$$

Equations $\langle 11\rangle$ and $\langle 13\rangle$ describe a relation similar to the risk-neutrality relation $\langle 1\rangle$, but with different probabilities. Using equations $\langle 11\rangle-$ $\langle 13\rangle$ and the terminal values of $V$ given in equation $\langle 8\rangle$, option prices can be calculated. Hence, we indeed have a simple binomial model to price floating strike currency lookback call options.

In Table 1 the values for a European currency lookback call option are given for several values of $\sigma$ and $N$, with $S=100, \tilde{r}_{d}=0.04, \tilde{r}_{f}=0.07$ and $T=0.5$. Also, the analytical value calculated using Conze and Viswanathan (1991) is given. At first sight, the binomial values in Table 1 seem to converge very slowly to the analytical one. But in the discrete model, the minimum is taken over a finite number of points, while the analytical value is calculated over infinitely many points. With finitely many observations, the option value should always be lower than with infinitely many observations. In reality, contracts always have finitely many observation points, such as daily, or even weekly fixings. Hence, the difference in value between an $N$-period and a continuous model should be split into two parts. The difference caused by a lower number of observations and the approximation error. This issue is thoroughly studied in the next section.

Next, binomial models for fixed strike currency lookback options are derived. The payoff of a European fixed strike currency lookback call with exercise price $K$ is given by

$$
\operatorname{Max}\left[\operatorname{Max}_{0 \leq i \leq N} S\left(t_{i}\right)-K, 0\right] .
$$

The value of this option at time $t_{j}$ once again depends not only on $t_{j}$ and $S\left(t_{j}\right)$, 
TABLE 1. European floating strike currency lookback call $\left(S=100, \tilde{r}_{d}=0.04, \tilde{r}_{f}=0.07\right.$ and $T=0.5$ )

\begin{tabular}{rccc}
\hline$N$ & \multicolumn{3}{c}{ Option price } \\
\cline { 2 - 4 } & $\sigma=0.1$ & $\sigma=0.2$ & $\sigma=0.3$ \\
\hline 50 & 4.24 & 8.97 & 13.52 \\
100 & 4.37 & 9.20 & 13.85 \\
500 & 4.54 & 9.52 & 14.31 \\
1000 & 4.58 & 9.60 & 14.42 \\
5000 & 4.63 & 9.71 & 14.57 \\
10000 & 4.65 & 9.73 & 14.60 \\
Analytic & 4.68 & 9.79 & 14.69 \\
\hline
\end{tabular}

but also on $\bar{M}\left(t_{j}\right)$. Two cases must be distinguished. If $\bar{M}\left(t_{j}\right) \leq K$ then $\bar{M}\left(t_{j}\right)$ has no effect on the final option payoff. Hence, $\bar{M}\left(t_{j}\right)$ doesn't matter in this case. If $\bar{M}\left(t_{j}\right)>K$, the option holder is ascertained of a payoff equal to $\bar{M}\left(t_{j}\right)-K$ at maturity. In addition, he or she also holds a similar option with an exercise price equal to $\bar{M}\left(t_{j}\right)$. Although, the option price depends on $\bar{M}\left(t_{j}\right)$, the option can be split in two parts. A known payoff at maturity equal to

$$
\operatorname{Max}\left(\bar{M}\left(t_{j}\right)-K, 0\right) \text {, }
$$

and a European fixed strike currency lookback call option with an exercise price equal to

$$
K^{\prime}=\operatorname{Max}\left(\bar{M}\left(t_{j}\right), K\right) .
$$

Only for this last part the option value has to be calculated. The first part has a present value equal to $\operatorname{Max}\left(\bar{M}\left(t_{j}\right)-K, 0\right) / r^{N-j}$. In this way, the option valuation problem is reduced to options with a maximum from the past below or equal to the strike price. The strike price is no longer fixed but changes over time.

From now on it is assumed that $K^{\prime}$ is equal to $S u^{m}$ for some integer $m$. If $K^{\prime}$ is not the initial strike price, this is always true. In the other case it is not a severe restriction if $N$ is large enough. As in the floating strike case, the state variable is defined by

$$
k=\ln \left[S\left(t_{j}\right) / K^{\prime}\right] / \ln (u) \text {. }
$$

This number is always non-positive. Let $C_{X}\left(S\left(t_{j}\right), K^{\prime}, t_{j}\right)$ be the value of the fixed strike currency lookback call option with $S\left(t_{j}\right) \leq K^{\prime}$. We claim that $C_{X}$ can be written as

$$
C_{X}\left(S\left(t_{j}\right), K^{\prime}, t_{j}\right)=S\left(t_{j}\right) X\left(k, t_{j}\right),
$$

for some function $X\left(k, t_{j}\right)$ that depends on $k$ and $t_{j}$ only. Once again, the claim can be proved by backwards induction. At maturity, the option value is zero since $S\left(t_{j}\right) \leq K^{\prime}$. Hence the claim clearly holds with $X\left(k, t_{N}\right)=0$ for all $k$. 
Figure 3 describes the tree of possible movements from one state to another. For $k \leq-1$, the movements are pretty obvious. This also holds for $k=0$ and a downward move of the underlying exchange rate. For $k=0$, i.e. the exchange rate is equal to the strike, and an upward move, the new exchange rate exceeds the strike. In this case, the option holder is entitled to an extra payoff equal to $S\left(t_{j}\right) u-K^{\prime}$ at maturity.

Since $K^{\prime}=S\left(t_{j}\right)$, the present value of this payment is $S\left(t_{j}\right)\left[(u-1) / r^{N-j}\right]$ which is homogeneous in $S\left(t_{j}\right)$. After this upward move the new strike price is set equal to $S\left(t_{j}\right) u=K^{\prime} u$ and the state remains 0 . This explains the horizontal lines in Figure 3.

Using the same argument as in the previous case, the claim for $k \leq-1$ can be proved by backward induction, with $X\left(k, t_{j}\right)$ defined as

$$
X\left(k, t_{j}\right)=\left[q X\left(k+1, t_{j+1}\right)+(1-q) X\left(k-1, t_{j+1}\right)\right] \frac{\mu}{r} .
$$

For $k=0$, the defining relation for $X\left(0, t_{j}\right)$ is

$$
X\left(0, t_{j}\right)=\left[q X\left(0, t_{j+1}\right)+(1-q) X\left(-1, t_{j+1}\right)\right] \frac{\mu}{r}+p(u-1) / r^{N-j}
$$

This relation holds since the present value of the extra payment due to the increase of the exercise price is equal to the exchange rate multiplied by a function that depends on $u$ and $t_{j}$ only, i.e. $S\left(t_{j}\right)(u-1) / r^{N-j}$. Without the term $p(u-1) / r^{N-j}$, the equations $\langle 19\rangle$ and $\langle 20\rangle$ and the final condition would give option values identically zero. Due to this term there are positive option values. The tree starts at point $(0,0)$. Of course, it is also possible to start the tree at a point $(k, 0)$ with $k<0$, indicating the exchange rate is below the strike.

In Table 2 values of European fixed strike currency lookback call options are given for several values of $N$ and $\sigma$, with $S=100, Z=100, \tilde{r}_{d}=0.04, \tilde{r}_{f}=0.07$ and $T=0.5$. Once again the values depend heavily on the number of discrete time points, which is studied in next section.

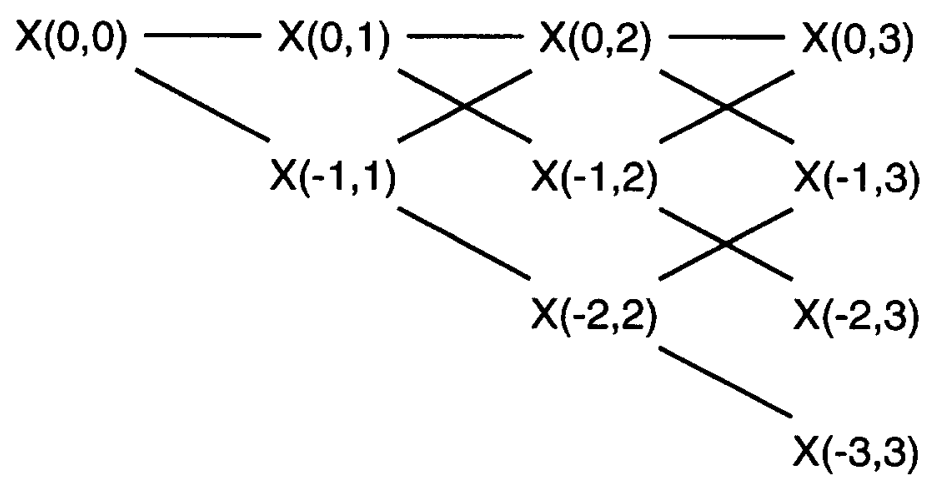

FIGURE 3. Fixed strike currency lookback call. 
TABLE 2. European fixed strike currency lookback call ( $S=100, K=100, \tilde{r}_{d}=0.04, \tilde{r}_{f}=0.07$ and $T=0.5$ )

\begin{tabular}{rccc}
\hline \multirow{2}{*}{$N$} & \multicolumn{3}{c}{ Option price } \\
\cline { 2 - 4 } & $\sigma=0.1$ & $\sigma=0.2$ & $\sigma=0.3$ \\
\hline 50 & 6.20 & 11.51 & 17.05 \\
100 & 6.34 & 11.80 & 17.50 \\
500 & 6.53 & 12.20 & 18.14 \\
1000 & 6.57 & 12.30 & 18.30 \\
5000 & 6.64 & 12.44 & 18.51 \\
10000 & 6.65 & 14.47 & 18.56 \\
Analytic & 6.78 & 12.70 & 18.92 \\
\hline
\end{tabular}

\section{The observation frequency}

As remarked in the previous section, Tables 1 and 2 suggest that the models seem to converge very slowly to the analytical values. This might be explained by the difference in the number of points in time the underlying exchange rate is observed. In reality, exchange rates are only observed at a finite number of points in time.

Consider a European floating strike currency lookback call with time to expiration equal to half a year. If the contract uses weekly fixings to calculate the minimum, there are only 26 observation points. Hence, the values for $N>26$ might be too high. On the other hand, the binomial model with 26 periods is very rough since it assumes the exchange rate can move to only two different rates within a week. To solve this problem the models of the previous section are extended to allow a distinction between observation and nonobservation points, or fixings and non-fixings.

Let $t_{i}, i=0,1, \ldots, N$, be discrete points in time of the binomial model. Let $t_{l}, 1=0, Z, 2 Z, \ldots, L Z=N$, be the observation points. Hence, between two subsequent fixings there are $Z-1$ other points. Define

$$
\underline{M}^{Z}\left(t_{j}\right)=\min _{0 \leq i Z \leq j} S\left(t_{i Z}\right)
$$

and

$$
k=\ln \left[S\left(t_{j}\right) / \underline{M}^{Z}\left(t_{j}\right)\right] / \ln (u) .
$$

Now $k$ is an integer larger than $-Z$.

Let $C^{Z}\left(S\left(t_{j}\right), M^{Z}\left(t_{j}\right), t_{j}\right)$ be the value of a floating strike currency lookback call option, of which the underlying is observed at the points $t_{i z}$ only. Once again we claim and prove that

$$
C^{Z}\left(S\left(t_{j}\right), \underline{M}^{Z}\left(t_{j}\right), t_{j}\right)=S\left(t_{j}\right) V^{Z}\left(k, t_{j}\right),
$$

for some function $V^{Z}$ depending only on $k$ and $t_{i}$.

In Figure 4, a tree with $Z=3$ is constructed. The parallel lines from $V^{3}(-2$, 


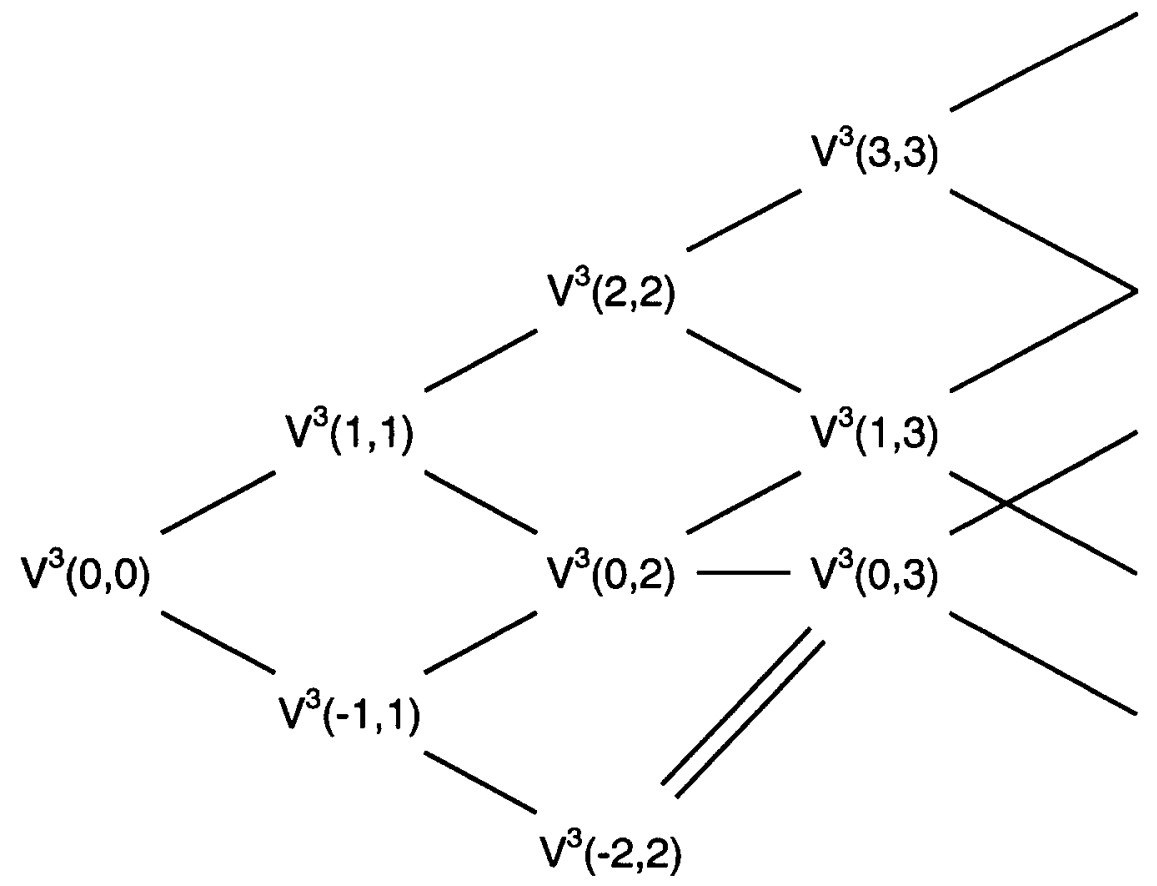

FIGURE 4. Floating strike currency lookback call, with $Z=3$.

$\left.t_{2}\right)$ to $V^{3}\left(0, t_{3}\right)$ need some clarification, while all the other lines are similar to the previous case. In $V^{3}\left(-2, t_{2}\right), S\left(t_{2}\right)=M^{3}\left(t_{2}\right) \cdot u^{-2}$. With either an upward or a downward move, the new exchange rate will be below $M^{3}\left(t_{2}\right)$. Hence, the new exchange rate will become the new minimum $M^{3}\left(t_{3}\right)$. Thus, the new state will be 0 . The recurrence relations for $V^{Z}\left(k, t_{j}\right)$ are given by $\langle 11\rangle$ for $k \geq 1$ and/or $t_{j+1} \neq i Z$ for all $i$. In the other cases we have

$$
V^{Z}\left(0, t_{i Z-1}\right)=\left[q V^{Z}\left(1, t_{i Z}\right)+(1-q) V^{Z}\left(0, t_{i Z}\right)\right] \frac{\mu}{r},
$$

and

$$
V^{Z}\left(k, t_{i Z-1}\right)=\left[q V^{Z}\left(0, t_{i Z}\right)+(1-q) V^{Z}\left(0, t_{i Z}\right)\right] \frac{\mu}{r}=V^{Z}\left(0, t_{i Z}\right) \frac{\mu}{r},
$$

for $k \leq-1$. The values at maturity, $V^{Z}\left(k, t_{L Z}\right)$, are again given by (9).

Hence, $\langle 9\rangle,\langle 11\rangle$ and $\langle 23\rangle-\langle 25\rangle$ allow us to calculate option prices with more periods in the tree than there are fixings.

In Table 3 European floating strike currency lookback call option values are given for different values of $L$ and $Z$ for the option in Table 1 with $\sigma=0.2$. As all points in the first column of Table 3 are observation points, the prices there are calculated in exactly the same way as those in the second column of Table 1. The columns in Table 3 show that the option value increases with the number of observations. Within each row, the number of fixings is kept 
TABLE 3. European floating strike currency lookback call, with different $L$ and $Z(S=100$, $\tilde{r}_{d}=0.04, \tilde{r}_{f}=0.07, \sigma=0.02$ and $T=0.5$ )

\begin{tabular}{|c|c|c|c|c|c|c|c|c|}
\hline \multirow[t]{2}{*}{$L$} & \multicolumn{8}{|c|}{$Z$} \\
\hline & 1 & 10 & 50 & 100 & 200 & 500 & 1000 & 2000 \\
\hline 1 & 6.14 & 4.65 & 4.76 & 4.77 & 4.78 & 4.78 & 4.78 & 4.78 \\
\hline 2 & 6.45 & 5.72 & 5.82 & 5.83 & 5.83 & 5.84 & 5.84 & 5.84 \\
\hline 6 & 7.65 & 7.51 & 7.23 & 7.24 & 7.24 & 7.25 & 7.25 & 7.25 \\
\hline 26 & 8.68 & 8.42 & 8.46 & 8.47 & 8.47 & 8.47 & 8.48 & 8.48 \\
\hline 125 & 9.26 & 9.14 & 9.16 & 9.17 & 9.17 & 9.17 & 9.17 & 9.17 \\
\hline
\end{tabular}

constant, while the number of nodes between observations varies. The rows converge, and for $Z=100$ the option values are already quite accurate. Convergence depends not only on the number of periods between two fixings but also on the total number of periods. Hence, with daily fixings we need less periods between observations than with weekly fixings. It can also be concluded that the analytical models give lousy approximations even if daily fixings ( $L=125$ ) are used. The daily fixing option price is only 9.17 , compared to the analytical value of 9.79 . Hence, the slow convergence in Table 1 is largely due to the different number of observations, and is not a weakness of the model. Finally, there is a substantial price difference between daily and weekly fixing options (9.17 vs 8.48 ).

Also for fixed strike currency lookback options, non-observation points can be introduced. This is described in Figure 5 for $Z=3$, where $k$ again measures the difference between the actual exchange rate and the strike price in powers of $u$. The backward recurrence relations are given by $\langle 19\rangle$ for $k \leq-1$ and/or $t_{j+1} \neq i Z$ for all $i$. In the other cases the following relations can easily be verified to hold:

$$
\begin{aligned}
X^{Z}\left(0, t_{i Z-1}\right)= & {\left[q X^{Z}\left(0, t_{i Z}\right)+(1-q) X^{Z}\left(-1, t_{i Z}\right)\right] \frac{\mu}{r} } \\
& +p(u-1) / r^{N-i Z+1}
\end{aligned}
$$

and

$$
\begin{aligned}
X^{Z}\left(k, t_{i Z-1}\right)= & X^{Z}\left(0 t_{i Z}\right)+\left[p\left(u-u^{-k}\right)\right. \\
& \left.+(1-p)\left(u^{-1}-u^{-k}\right)\right] / r^{N-i Z+1},
\end{aligned}
$$

for $k \geq 1$. In Table 4 values of European fixed strike currency lookback call options are given when there are observation and non-observation points in the tree. Table 4 should be compared with Table 2 in the same way as Table 3 was compared with Table 1 . The convergence depends not only on the number of discrete time points between two observations, but also on the total number of nodes. Hence, with daily observations we need less discrete time points between observations than with monthly fixings. Nevertheless, for daily observations the total number of nodes in the tree needs to be larger. It is also clear 
Currency lookback options and observation frequency: THF Cheuk and TCF Vorst

TABLE 4. European fixed strike currency lookback call, with different $L$ and $Z$ ( $S=100$, $K=100, \tilde{r}_{d}=0.04, \tilde{r}_{f}=0.07, \sigma=0.2$ and $T=0.5$ )

\begin{tabular}{rrrrrrrrr}
\hline$L$ & \multicolumn{10}{c}{$Z$} & \multicolumn{1}{c}{$Z$} & \\
\cline { 2 - 9 } & \multicolumn{1}{c}{1} & \multicolumn{1}{c}{10} & \multicolumn{1}{c}{50} & 100 & 200 & 500 & 1000 & 2000 \\
\hline 1 & 7.98 & 6.29 & 6.38 & 6.39 & 6.39 & 6.40 & 6.40 & 6.40 \\
2 & 8.50 & 7.54 & 7.64 & 7.65 & 7.65 & 7.66 & 7.66 & 7.66 \\
6 & 9.90 & 9.27 & 9.35 & 9.36 & 9.36 & 9.36 & 9.37 & 9.37 \\
26 & 11.15 & 10.83 & 10.87 & 10.88 & 10.88 & 10.89 & 10.89 & 10.89 \\
125 & 11.88 & 11.73 & 11.75 & 11.76 & 11.76 & 11.76 & 11.76 & 11.76 \\
\hline
\end{tabular}

that the number of observations is a very important parameter for the value of a fixed strike currency lookback call option. The weak convergence in Table 2 should be attributed mainly to this parameter and is not a flaw of the model.

\section{American options}

The binomial models for European floating strike currency lookback options described in the previous section can easily be extended for the American type. For simplicity, we first consider the model of Section I where all discrete time points are observation points. When the American floating strike currency lookback call option is exercised early, the payoff is equal to

$$
S\left(t_{j}\right)-\min _{0 \leq i \leq j} S\left(t_{j}\right)=S\left(t_{j}\right)\left(1-\underline{M}\left(t_{j}\right) / S\left(t_{j}\right)\right)=S\left(t_{j}\right)\left(1-u^{-k}\right) .
$$

Hence, the option should be exercised early if and only if $1-u^{-k}>V\left(k, t_{j}\right)$. To calculate American option values the standard procedure should be used. Check in equation $\langle 11\rangle$ whether the right hand side is less than $1-u^{-k}$. If so, replace the right hand side by this expression. The same kind of trick can be applied to the models with non-observation points as in Section II.

In Table 5 values for American currency lookback call options are given,

TABLE 5. American floating strike currency lookback call, with different $L$ and $Z(S=100$, $\tilde{r}_{d}=0.04, \tilde{r}_{f}=0.07, \sigma=0.2$ and $T=0.5$ )

\begin{tabular}{|c|c|c|c|c|c|c|c|c|}
\hline \multirow[t]{2}{*}{$L$} & \multicolumn{8}{|c|}{$Z$} \\
\hline & 1 & 10 & 50 & 100 & 200 & 500 & 1000 & 2000 \\
\hline 1 & 6.14 & 4.87 & 4.93 & 4.94 & 4.94 & 4.94 & 4.94 & 4.94 \\
\hline 2 & 6.85 & 5.93 & 6.01 & 6.01 & 6.02 & 6.02 & 6.02 & 6.02 \\
\hline 6 & 7.98 & 7.38 & 7.45 & 7.46 & 7.46 & 7.46 & 7.46 & 7.46 \\
\hline 26 & 8.95 & 8.67 & 8.71 & 8.71 & 8.71 & 8.72 & 8.72 & 8.72 \\
\hline 125 & 9.52 & 9.40 & 9.42 & 9.42 & 9.42 & 9.42 & 9.42 & 9.42 \\
\hline
\end{tabular}




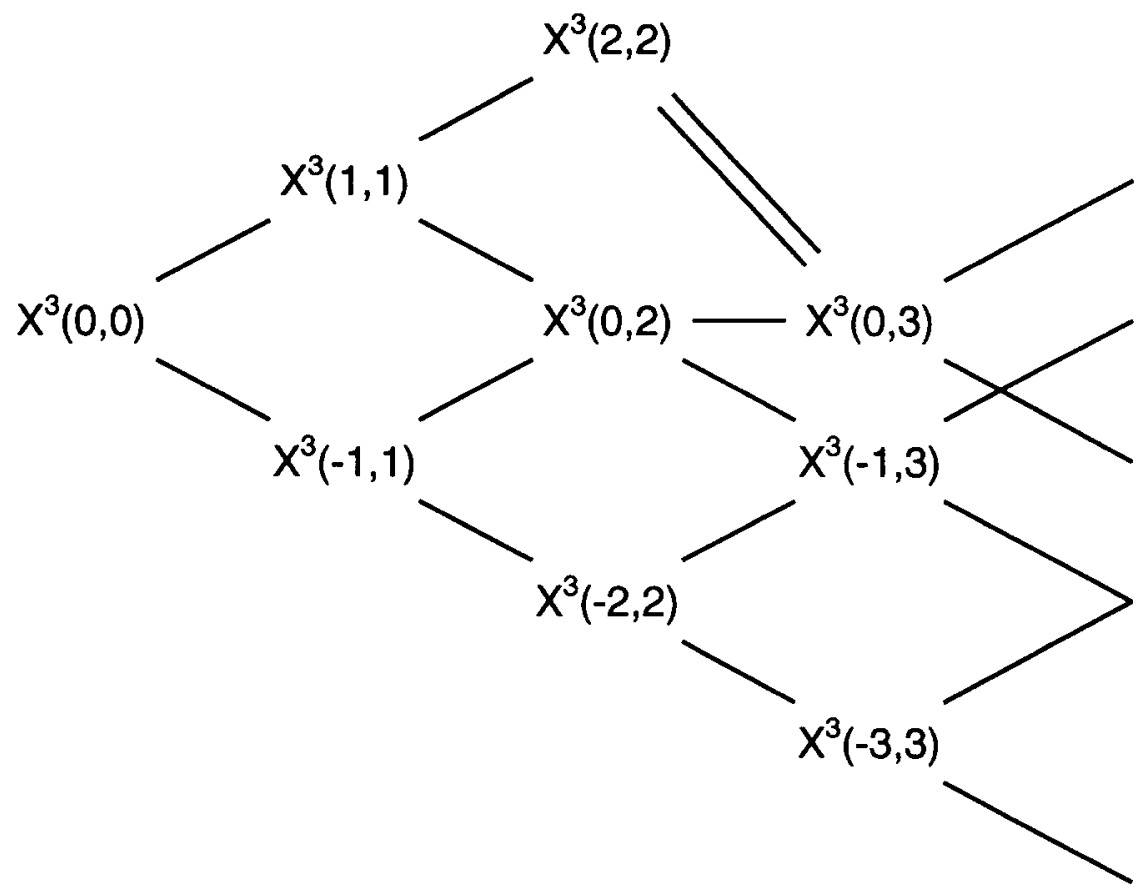

FIGURE 5. Fixed strike currency lookback call, with $Z=3$.

using models with non-observation points. Again, the model converges with $Z=100$, and the prices depend heavily on the number of fixings.

Unfortunately, the binomial model for the European fixed strike currency lookback option cannot be adjusted to price its American variant.

\section{Comparison to other methods}

There are alternative methods proposed by other authors to value lookback options using a lattice approach. Hull and White (1993) describe a general approach to value path dependent options. This approach can also be applied to lookback options. They construct the standard binomial tree in which each singular node is subdivided into different states according to the value of the sufficient statistic, which is for a floating strike lookback call equal to the minimum observed up to that moment in time. For each state within a node they calculate the option value from specific states in nodes one period ahead. With the same number of steps in the tree, they produce exactly the same values as the method described in this paper. Hence, both procedures have the same convergence. However, for a given number of steps in the tree our method is much faster since we essentially have only one state in each node while they have on average a number of states that increases linearly in $n$. Hence, the computational time for our method is a quadratic function of the number of steps, while their method is a cubic function. 
Babbs (1992) has also proposed a valuation method for floating strike lookback options but not for fixed strikes. However, his method is derived using continuous-time mathematics and based on the reflection theory of Brownian motions. Due to its simplicity, our method is accessible to a much wider audience. To do numerical calculations Babbs uses lattices that are similar to Figures 2 and 4 . If we take the important issue of the observation frequency into account, the method of Babbs has the same convergence properties as our method. For continuous-time observation floating strike lookbacks, the method of Babbs converges faster. Essentially Babbs doesn't allow for the horizontal lines in Figure 2, but assumes that there is a reflecting barrier, i.e. both lines from $V(0, k)$ go to $V(1, k+1)$. This is due to the fact that a Brownian motion doesn't have any time interval over which it is monotonically decreasing. In each interval, whatever its length, the motion goes up and down. Hence, there is no interval on which the variable $V$ stays at zero, but it always bounces away from zero. Therefore, to approximate the analytic value, Babbs' method converges faster since it takes this bouncing away from zero into account. In reality, however, the exchange rate could continue to drop for a certain period in time. Hence our method might be a realistic alternative for Babbs' method. For discretely observed lookbacks this peculiar property of the Brownian motion is not relevant and hence both methods have the same convergence. Cheuk and Vorst (1996) describe a similar difference in valuation between continuously and discretely observed barrier options. Also here the difference is due to a peculiar property of the theoretical Brownian motion. Kat (1995) has compared the method of this paper with those of Babbs and Hull and White for discretely observed lookbacks. His conclusions concerning convergence confirm the remarks made above.

\section{Conclusion}

In this paper binomial models for currency lookback options have been developed. These models have the following three important properties. First, they have only one state variable, which allows efficient and fast computation of option values. Second, the models are derived using the simple Cox, Ross and Rubinstein arbitrage arguments alone, without the need of the theory of Brownian motions. Hence, the models are easy to understand. Finally, these models allow us to consider the influence of the observation frequency on option prices. This influence is shown to be substantial and the analytical formulas based on a continuous observation of the underlying exchange rate give poor approximations for real-life option prices. Especially this last feature should be the compelling reason to use the binomial model instead of the analytical formula, just as Cox, Ross and Rubinstein is used instead of Black-Scholes to value American options.

\section{Notes}

1. In the figures, $V(k, j)$ is used for $V\left(k, t_{j}\right)$. This change in notation is also used in the other figures. 


\section{References}

Babbs, S. (1992) Binomial valuation of lookback options. Working Paper, Midland Global Markets.

Black, F. and Scholes, M. (1973) The pricing of options on corporate liabilities. Journal of Political Economy, 81, 637-654.

Cheuk, T. H. F. and Vorst, T. C. F. (1996) Complex barrier options. Journal of Derivatives, 4(1), 8-22.

Goldman, M. B., Sossin, H. B. and Gatto, M. A. (1979) Path dependent options: buy at the low, sell at the high. Journal of Finance, 34, 1111-1127.

Conze, A. and Viswanathan (1991) Path dependent options, the case of lookback options. Journal of Finance, 46, 1893-1907.

Cox, J. C. and Ross, S. A. (1976) The valuation of options for alternative stochastic processes. Joumal of Financial Economics, 3, 145-166.

Cox, J. C., Ross, S. A. and Rubinstein, M. (1979) Option pricing: a simplified approach. Journal of Financial Economics, 7, 229-263.

Hull, J. and White, A. (1993) Efficient procedures for valuing European and American path-dependent options. Journal of Derivatives, 1(1), 21-31.

Kat, H. M. (1995) Pricing lookback options using binomial trees: an evaluation. Journal of Financial Engineering, 4, 375-397. 